\title{
The Profile of Timing Dialysis Initiation in Patients with End-stage Renal Disease in China: A Cohort Study
}

\author{
Ying Liu ${ }^{a, b}$ Luping Wang ${ }^{b}$ Xianfeng Han ${ }^{b}$ Yang Wang ${ }^{c}$ Xuefeng Sun ${ }^{d}$ \\ Hongli Jiang e Wei Shi ${ }^{f}$ Wenhu Liug ${ }^{g}$ Ping Fu $^{\text {h }}$ Xiaoqiang Ding ${ }^{i}$ \\ Hongli Lin ${ }^{a, b}$ Xiangmei Chen ${ }^{d}$ on behalf of the DIFE Study Group \\ ${ }^{a}$ Graduate School of Dalian Medical University, Dalian, China; ${ }^{b}$ Department of Nephrology, \\ The First Affiliated Hospital, Institute for Nephrology Research of Dalian Medical University, \\ Center for Kidney Diseases Translational Medicine of Liaoning Province, Dalian, China; \\ 'Medical Research and Biometrics Center, Cardiovascular Institute and Fuwai Hospital, \\ National Center for Cardiovascular Diseases, Chinese Academy of Medical Sciences and \\ Peking Union Medical College, Beijing, China; ${ }^{\mathrm{d}}$ Department of Nephrology, Chinese \\ PLA General Hospital, National Clinical Research Center for Kidney Diseases, State Key \\ Laboratory of Kidney Diseases, Chinese PLA Institute of Nephrology, Beijing, China; ${ }^{\mathrm{e}} \mathrm{Blood}$ \\ Purification Center, The First Affiliated Hospital of Xi'an Jiaotong University, Xi'an, China; \\ ${ }^{f}$ Division of Nephrology, Guangdong General Hospital, Guangdong Academy of Medical \\ Sciences, Guangzhou, China; 'Division of Nephrology, Beijing Friendship Hospital, Capital

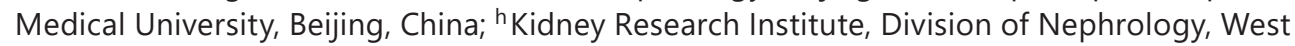 \\ China Hospital of Sichuan University, Chengdu, China; 'Division of Nephrology, Zhongshan \\ Hospital, Fudan University, Shanghai, China
}

\section{Keywords}

End-stage renal disease $\cdot$ Hemodialysis - Timing of dialysis initiation · Glomerular filtration rate Mortality

\begin{abstract}
Background: Hemodialysis is the main approach for renal replacement therapy in patients with end-stage renal disease (ESRD) in China. The timing of dialysis initiation is one of the key factors influencing patient survival and prognosis. Over the past decade, the relationship between the timing of dialysis initiation and mortality has remained unclear in patients with ESRD in China. Methods: Patients who commenced maintenance hemodialysis from 2009 to 2014 from 24 hemodialysis centers in Mainland China were enrolled in the study $(n=1,674)$. Patients were divided into 2 groups based on the year they started hemodialysis (patients who
\end{abstract}

Y.L. and L.W. contributed equally to this work.

A complete list of investigators comprising the Dialysis Initiation based on Fuzzy mathematics Equation (DIFE) study group is provided in the Appendix.

Dr. Hongli Lin

Department of Nephrology

The First Affiliated Hospital of Dalian Medical University

No. 222, Zhongshan Road, Dalian 116011 (China)

E-Mail hllin@dlmedu.edu.cn
Dr. Xiangmei Chen

Department of Nephrology, General Hospital of PLA

Fuxing Road 28

Beijing 100853 (China)

E-Mail xmchen301@126.com 


\section{Kidney \\ Blood Pressure \\ Research}

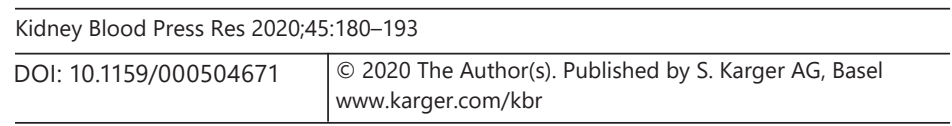

Liu et al.: Timing of Dialysis Initiation in ESRD Patients in China

started hemodialysis from 2009 to 2011, and patients who started hemodialysis from 2012 to 2014). Analysis of the yearly change in the estimated glomerular filtration rate (eGFR) at the initiation of dialysis was performed for the 2 groups. Meanwhile, the patients were divided into 3 groups based on their eGFR at the initiation of dialysis $(<4,4-8$, and $>8 \mathrm{~mL} / \mathrm{min} / 1.73$ $\mathrm{m}^{2}$ ). For these 3 groups, the relationship between the eGFR at the start of dialysis and mortality were analyzed. Results: The average eGFRs were 5.68 and $5.94 \mathrm{~mL} / \mathrm{min} / 1.73 \mathrm{~m}^{2}$ for 20092011 and 2012-2014, respectively. Compared with the 2009-2011 group, the proportion of patients with diabetes in 2012-2014 increased from 26.7 to $37.7 \%$. The prognosis of patients with different eGFRs at the start of dialysis was analyzed using Kaplan-Meier survival curves. After adjusting for confounding factors through a Cox regression model, no significant difference was demonstrated among the 3 groups $\left(<4 \mathrm{~mL} / \mathrm{min} / 1.73 \mathrm{~m}^{2}\right.$ was used as the reference, in comparison with $4-8 \mathrm{~mL} / \mathrm{min} / 1.73 \mathrm{~m}^{2}[p=0.681]$ and $>8 \mathrm{~mL} / \mathrm{min} / 1.73 \mathrm{~m}^{2}[p=0.403]$ ). Conclusion: In Mainland China, the eGFR at the start of dialysis did not change significantly over time from 2008 to 2014 and had no association with the mortality of patients with ESRD.

(C) 2020 The Author(s)

Published by S. Karger AG, Basel

\section{Introduction}

In China, hemodialysis is the main approach for renal replacement therapy for patients with end-stage renal disease (ESRD). Among all patients undergoing dialysis, $90 \%$ of them received hemodialysis $[1,2]$. The optimal timing of dialysis initiation could improve a patient's quality of life and prognosis [3-5]. However, controversy remains regarding when to initiate hemodialysis. In 1985, Bonomini et al. [6] reported that initiation of dialysis in patients with a high glomerular filtration rate (GFR; estimated GFR [eGFR]) could reduce the hospitalization rate and mortality. Since then, several studies have suggested that dialysis at higher eGFRs could improve patients' prognosis by reducing the incidence of malnutrition and hospitalization rates $[7,8]$. In clinical practice, initiation of dialysis at high eGFRs is called "early dialysis." However, in the last 10 years, many studies have suggested that early dialysis did not benefit patients and could even increase the risk of death [9-13].

Since the 1990s, early dialysis was preferred in the United States, Canada, and Europe, and the proportion of patients receiving dialysis with an eGFR $>10 \mathrm{~mL} / \mathrm{min} / 1.73 \mathrm{~m}^{2}$ had been increasing until 2010, after which it decreased [14]. In comparison, in Asia (Japan, South Korea, and Singapore), patients with ESRD began to undergo dialysis with a relatively lower eGFR (average value approximately $5 \mathrm{~mL} / \mathrm{min} / 1.73 \mathrm{~m}^{2}$ ) [15-17]. Based on these findings, many countries and regions have decided upon an appropriate time to start hemodialysis for their own patients [18-21]. A cross-sectional survey of a nationally representative sample of Chinese adults demonstrated that 120 million patients have chronic kidney disease (CKD) with 19 million of them having stage 3 CKD [22], 110 million have diabetes [23] injury, and 250 million have hypertension [24]. It was estimated that if $30 \%$ of patients with stage 3 CKD develop to ESRD, $30 \%$ of patients with diabetes have kidney injury, and $50 \%$ of them develop ESRD within 10-20 years, there will be an annual increase of 0.5-1 million new ESRD patients over the next 10-20 years in China.

However, according to the Chinese Renal Data Service, there were 447,435 patients undergoing dialysis until 2016, with an increase of 75,831 cases, a prevalence of patients undergoing dialysis of 325 per million population (PMP), and an annual increase of approximately 50\% in 2016 [25]. In comparison, the prevalence of patients with ESRD on dialysis reached 3,093 PMP in 2016 in Taiwan, whereas it was 758 PMP and 1,498 PMP in Hong Kong and the United States, respectively. Therefore, the current proportion of patients with ESRD undergoing hemodialysis is still relatively low in China, which is mainly attributed to 


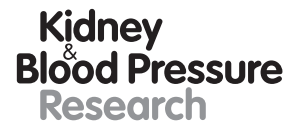

Kidney
Blood Pressure
Research

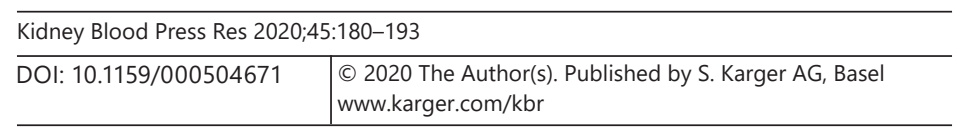

Liu et al.: Timing of Dialysis Initiation in ESRD Patients in China

the short survival time extended by hemodialysis. The average dialysis duration for patients who died in 2016 in China was 42.8 months, while the average dialysis duration for patients still on dialysis was 41 months [25]. Therefore, it is necessary to gain a clear insight into the start time of hemodialysis in Chinese patients, which will help to determine the optimal timing of dialysis initiation to improve the quality of life of dialysis patients and to reduce their mortality rate. However, in Mainland China, only Beijing and Shanghai have regional research reports on the time of dialysis initiation, while there is lack of such reports at the national level [26, 27]. Furthermore, as the dialysis initiation guidelines were updated by the European Renal Best Practice in 2011 [21] and Kidney Disease Improving Global Outcomes in 2012 [28], the changes in eGFR at initiation in China were unknown. Therefore, the present study, which is a nationwide multicenter retrospective cohort study, analyzed the overall trends and characteristics of the timing of dialysis initiation, the trend in eGFR at initiation before and after 2012, and evaluated the relationship between the timing of dialysis initiation and mortality in patients with ESRD in Mainland China. Meanwhile, the timing of dialysis initiation in Chinese patients with ESRD was also compared with that in other countries.

\section{Materials and Methods}

\section{Study Design and Patient Selection}

Twenty-four hemodialysis centers from Mainland China participated in this study (including the Northeast, North, East, Central south, Southwest, and Southeast; the list of the participating institution is included in the appendix). This study was approved by the Ethical Committee of the first affiliated hospital of Dalian Medical University (LUCK 2014-25) and Ethical Committees of all the participating institutions. We obtained written informed consent from all patients, and personal information was protected during data collection.

Screened patients comprised those with ESRD who started maintenance hemodialysis between January 1, 2009, and December 31, 2014, and met the following inclusion criteria: (1) 16-80 years old; (2) diagnosed with CKD with 2 consecutive eGFR measurements of $<30$ $\mathrm{mL} / \mathrm{min} / 1.73 \mathrm{~m}^{2}$ within 3 months before dialysis; and (3) duration of maintenance hemodialysis was $>3$ months. The exclusion conditions were (1) patients who have undergone peritoneal dialysis or kidney transplantation before or after hemodialysis; (2) patients with the presence of cancer, chronic infection, liver cirrhosis, or other diseases that can affect survival time at the initiation of hemodialysis; (3) patients who died from nondisease-related causes, such as traffic accidents and suicide; (4) patients with acute kidney disease; and (5) patients who underwent emergency hemodialysis because of acute pulmonary edema, hypokalemia, or acidosis.

\section{Outcome}

The survival time of the patients was measured in months and was calculated from the date of dialysis initiation to the date of their death or to the final follow-up date (September $30,2016)$. Information on a patient's death was extracted from the patient's medical records, and the cause of their death was diagnosed by internal medicine physicians.

\section{Grouping of the Patients}

First, the patients were divided into 2 groups according to the time of hemodialysis initiation in fiscal years: 2009-2011, which included patients who started dialysis between 20092011; and 2012-2014, which included patients who started dialysis between 2012 and 2014. The patients' eGFRs and clinical parameters at the start of dialysis were compared between 


\section{Kidney \\ Blood Pressure \\ Research}

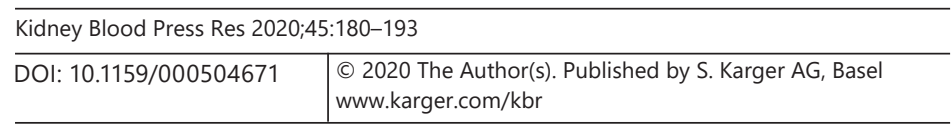

Liu et al.: Timing of Dialysis Initiation in ESRD Patients in China

the 2 groups. We also divided the cohort into 3 groups based on the patient's eGFR at the initiation of hemodialysis: $>8,4-8$, and $<4 \mathrm{~mL} / \mathrm{min} / 1.73 \mathrm{~m}^{2}$. The association between clinical factors and mortality for these 3 groups were analyzed.

\section{Data Collection}

Demographic data of the patients included gender, date of birth, and the date of dialysis initiation. Clinical data at the initiation of dialysis were collected including status of vascular pathways; presence of diabetes; clinical signs and/or symptoms including heart failure, digestive tract symptoms, edema of stage II and above, and uremia encephalopathy. The laboratory tests were performed within 3 months before dialysis initiation, and the tested factors included hemoglobin, serum albumin, serum urea nitrogen, serum creatinine, serum potassium, blood phosphate, and parathyroid hormone. Modification of diet in renal disease formula 7 was used to calculate the eGFR value at the initiation of dialysis in all patients. The formula is eGFR $=170 \times \mathrm{Scr}^{-0.999} \times \mathrm{age}^{-0.176} \times \mathrm{BUN}^{-0.170} \times \mathrm{Alb}^{0.318}(\times 0.762$, if the patient is female) [29], where Scr is serum creatinine, BUN is blood urea nitrogen, and Alb is albumin.

Data for all the patients were from electronic outpatient and inpatient medical records provided by the dialysis center. All researchers involved in this study were internal medical physicians and postgraduate students studying kidney disease, who have received unified training. The clinical symptoms and physical status of the patients at the start of dialysis, which were extracted from the patient's clinical records, were diagnosed by kidney disease physicians. Such data, which were checked by the data entry clerk when they were extracted, match the definition of clinical symptoms/signs of ESRD as defined in the Kidney Disease Improving Global Outcomes guidelines of 2015 [18]. In addition, to ensure the accuracy of data entry, data of 100 patients were randomly selected and entered independently by data entry clerks Y.L., L.W., and X.H., respectively.

\section{Statistical Analysis}

Continuous variables within a normal distribution were expressed as the mean \pm SD. Comparisons between groups were performed using an independent $t$ test, while comparisons between multiple groups were performed using single factor analysis of variance. Continuous variables not within a normal distribution were expressed as the median or 4-point spacing, and comparisons of these data between groups were performed using the Kruskal-Wallis test. Categorical variables were expressed as a percentage (\%), and differences between groups were analyzed using the $\chi^{2}$ test. A multiple imputation approach was applied to process missing data. Multivariate logistic regression analysis was performed to explore the association between prognosis and the clinical factors, including demographic data, clinical symptoms/signs, and laboratory results of various indicators at the start of dialysis. Kaplan-Meier survival curves, log-rank tests, and multivariate Cox regression models were used to analyze the relationship between the survival outcome after dialysis and the eGFR level at the start of dialysis. SPSS software (version 19.0, IBM Corp., Armonk, NY, USA) was used for statistical analysis. All statistical tests were bilateral, and $p<0.05$ was considered statistically significant.

\section{Results}

Cohort Characteristics

Between 2009 and 2014, there were 2,086 patients with ESRD who commenced maintenance dialysis in the 24 hemodialysis centers. Among these patients, 323 were excluded as they did not meet the inclusion criterions, and 89 were excluded because of lack of data on 


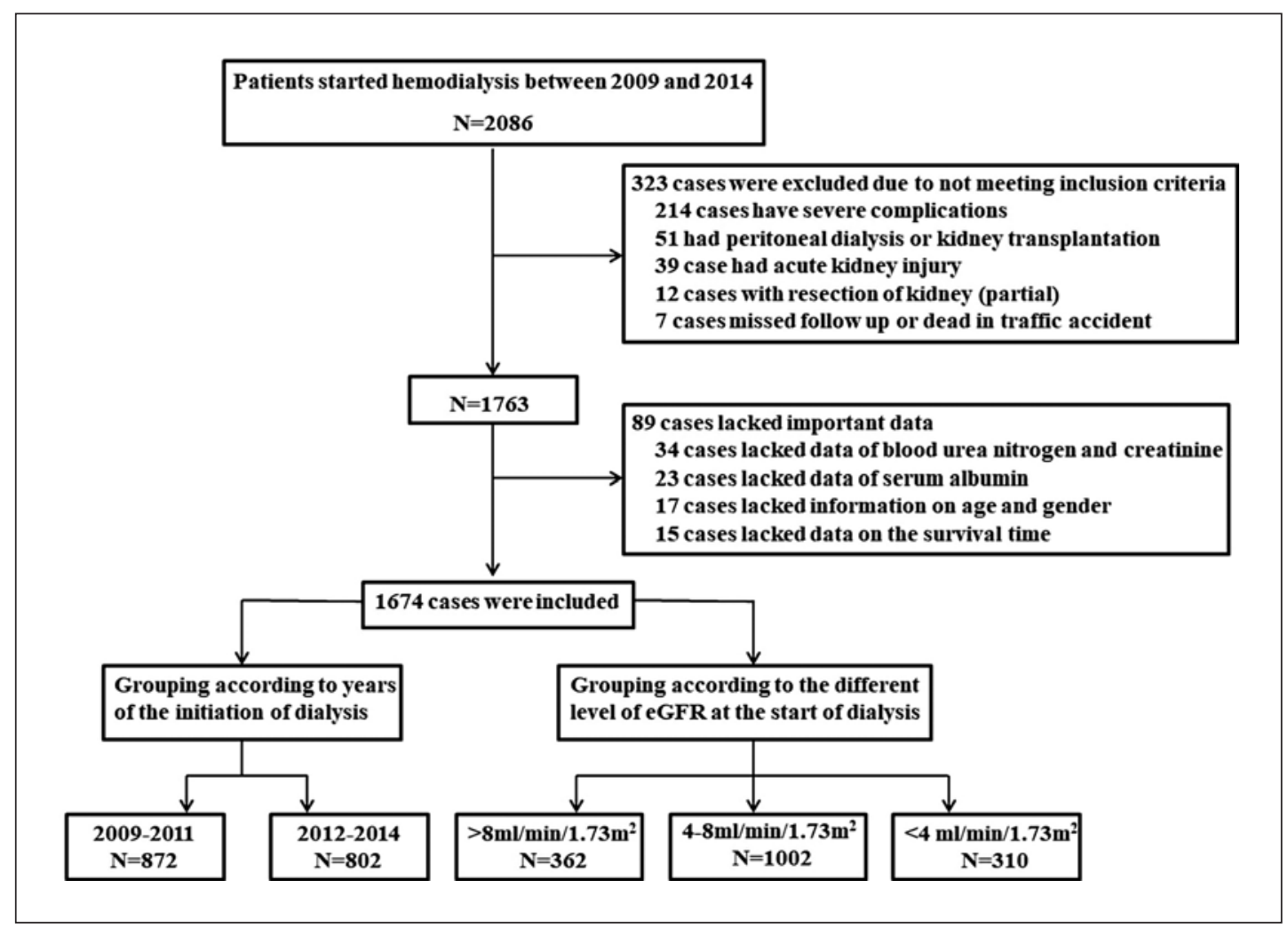

Fig. 1. Flow chart of the study. eGFR, estimated glomerular filtration rate.

the levels of urea nitrogen, creatinine, serum albumin, clinical signs and/or symptoms at the start of dialysis, or outcome data. Finally, a total of 1,674 patients were included in the study. The research flow chart is shown in Figure 1. For the selected patients, at the start of dialysis, male patients accounted for $61.9 \%$, patients with diabetes accounted for $31.9 \%$, the average age was $53.4 \pm 13.7$ years old, the average eGFR at the start of dialysis was $6.5 \pm 3.3 \mathrm{~mL} /$ $\mathrm{min} / 1.73 \mathrm{~m}^{2}$, and the median eGFR was $5.8 \mathrm{~mL} / \mathrm{min} / 1.73 \mathrm{~m}^{2}$.

\section{Comparison of Patient data between 2009-2011 and 2012-2014}

There were 872 patients who started dialysis between 2009 and 2011, and 802 patients who started dialysis between 2012 and 2014. There were no significant differences between the 2 groups regarding gender, age, and eGFR at the start of dialysis. Compared with the 2009-2011 group, the proportion of patients with diabetes and patients using central venous catheters in the 2012-2014 increased significantly from 26.7 to $37.7 \%$ and from 58.0 to 78.1 , respectively (both $p<0.001$ ). A comparison of the clinical signs and/or symptoms showed that there was a higher proportion of patients who had heart failure, symptoms of digestive tract disease, edema of stage II or above, or uremic encephalopathy in the 2009-2011 group compared with those in the $2012-2014$ group $(p<0.05)$. Mortality within 1 year after initiation is not significantly different between the 2 groups (Table 1 ).

Independent comparative analysis within the 2 groups showed that age and eGFR at the start of dialysis in patients with diabetes were higher than those in patients without diabetes. In addition, the proportion of patients with clinical signs and/or symptoms, including heart failure and edema at stage II or above, was also higher among patients with diabetes than in patients without diabetes. However, the levels of serum albumin and serum parathyroid 
Table 1. Baseline characteristics of patients who started dialysis in 2009-2011 and 2012-2014

\begin{tabular}{|c|c|c|c|}
\hline Variables & $\begin{array}{l}2009-2011 \\
(n=872)\end{array}$ & $\begin{array}{l}2012-2014 \\
(n=802)\end{array}$ & $p$ value \\
\hline Age, years, median (range $\left.{ }^{a}\right)$ & $55(45-63)$ & $55(45-63)$ & 0.494 \\
\hline Gender, male, $n(\%)$ & $536(61.5)$ & $536(61.5)$ & 0.712 \\
\hline Diabetes, $n(\%)$ & $233(26.7)$ & $302(37.7)$ & $<0.001$ \\
\hline \multicolumn{4}{|l|}{ Clinical signs and/or symptoms at the start of dialysis, $n(\%)$} \\
\hline Hear failure & $240(27.5)$ & $360(44.9)$ & $<0.001$ \\
\hline Symptoms of digestive tract & $231(26.5)$ & $343(42.8)$ & $<0.001$ \\
\hline Edema of stage II or above & $343(39.3)$ & $384(47.9)$ & $<0.001$ \\
\hline Uremia encephalopathy & $35(4.0)$ & $52(6.5)$ & 0.023 \\
\hline Vascular access at the start of dialysis, $n(\%)$ & & & $<0.001$ \\
\hline Central venous catheter & $506(58.0)$ & $626(78.1)$ & \\
\hline Arteriovenous fistula & $362(41.5)$ & $176(21.9)$ & \\
\hline Arteriovenous graft & $4(0.5)$ & $0(0)$ & \\
\hline eGFR at the start of dialysis, $\mathrm{mL} / \mathrm{min} / 1.73 \mathrm{~m}^{2}$, median (range) & $5.68(4.38-7.58)$ & $5.94(4.41-7.82)$ & 0.120 \\
\hline \multicolumn{4}{|l|}{ Laboratory tests, median (range) } \\
\hline Hemoglobin, g/L & $84.0(70.0-100.0)$ & $82.0(67.3-99.0)$ & 0.316 \\
\hline Serum albumin, $\mathrm{g} / \mathrm{L}$ & $36.0(31.0-39.3)$ & $35.3(30.8-38.8)$ & 0.743 \\
\hline Uric acid, $\mu \mathrm{mol} / \mathrm{L}$ & $462.0(376.3-538.8)$ & $453.5(374.2-548.0)$ & 0.484 \\
\hline Phosphorus, mmol/L & $1.91(1.55-2.33)$ & $1.92(1.56-2.40)$ & 0.222 \\
\hline Potassium, mmol/L & $4.73(4.16-5.30)$ & $4.78(4.30-5.40)$ & 0.045 \\
\hline Serum iPTH, $\mathrm{pg} / \mathrm{mL}$ & $225.3(125.1-434.6)$ & $264.9(127.8-426.0)$ & 0.030 \\
\hline Mortality within 1 year after dialysis initiation ${ }^{\mathrm{b}}, \% 0$ & 36.0 & 42.0 & 0.469 \\
\hline
\end{tabular}

${ }^{a}$ Ranges are the 25th-75th percentiles. ${ }^{b}$ Mortality rate reported as rate per 100 patient-years.

eGFR, estimated glomerular filtration rate; iPTH, immunoreactive parathyroid hormone.

hormone were significantly lower in patients with diabetes than in patients without diabetes. Further, mortality within 1 year after initiation was higher in patients with diabetes than in those without diabetes in 2012-2014, while there was no significant difference in the mortality rate between patients with and without diabetes for 2009-2011 (Table 2).

\section{Changes in the Timing of Dialysis Initiation between 2009 and 2014}

Calculation of the annual average eGFR at the start of dialysis demonstrated that the lowest value was $6.18 \mathrm{~mL} / \mathrm{min} / 1.73 \mathrm{~m}^{2}$ in 2009 , while the highest value was $6.80 \mathrm{~mL} /$ $\mathrm{min} / 1.73 \mathrm{~m}^{2}$ in 2014, with no significant change of the annual average eGFR at the start of dialysis ( $p=0.232$; Fig. 2 ). By comparing the eGFR at the start of dialysis between patients with diabetes and patients without diabetes in any year, we found that the eGFR in patients with diabetes from any year was higher than that in patients without diabetes (Fig. 2B). The proportion of patients with eGFRs of $<4 \mathrm{~mL} / \mathrm{min} / 1.73 \mathrm{~m}^{2}$, at $4-8 \mathrm{~mL} / \mathrm{min} / 1.73 \mathrm{~m}^{2}$ and $>8$ $\mathrm{mL} / \mathrm{min} / 1.73 \mathrm{~m}^{2}$ at the initiation of dialysis did not change significantly over the years $(p=$ 0.789; Fig. 2C). There was also no significant difference in the eGFR at the start of dialysis between 2009-2011 and 2012-2014 ( $p=0.627$; Fig. 2D).

\section{Factors Associated with the Timing of the Initiation of Hemodialysis}

Multivariate logistic regression analysis was performed for late start group, intermediate start group, and early start group. The results showed that being male, older, and having diabetes or heart failure before dialysis were the causes for early dialysis start. Meanwhile being female, having symptoms of digestive tract disease and hyperphosphatemia were associated with late dialysis start (Table 3). 
Kidney

Blood Pressure

Research
Kidney Blood Press Res 2020;45:180-193

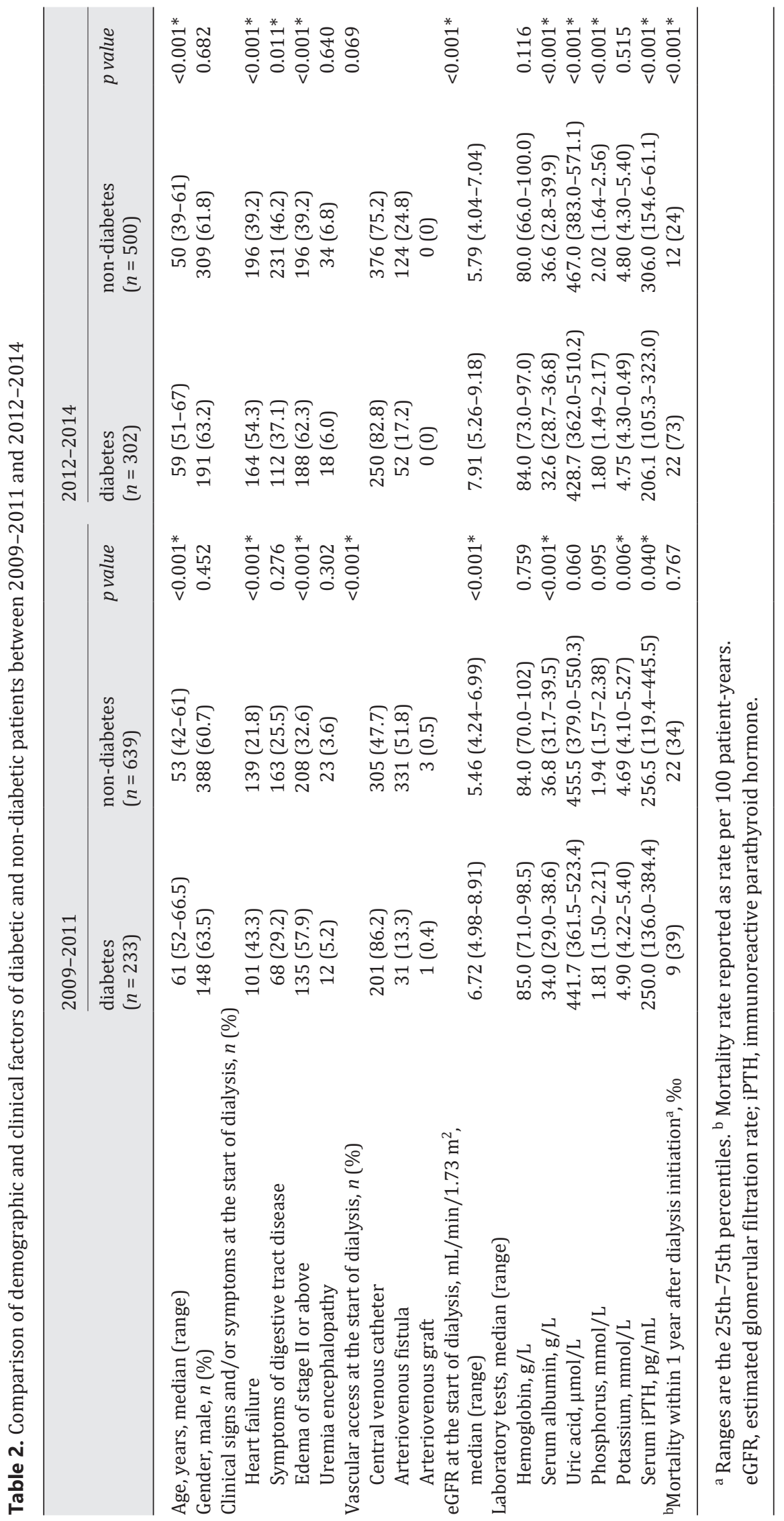




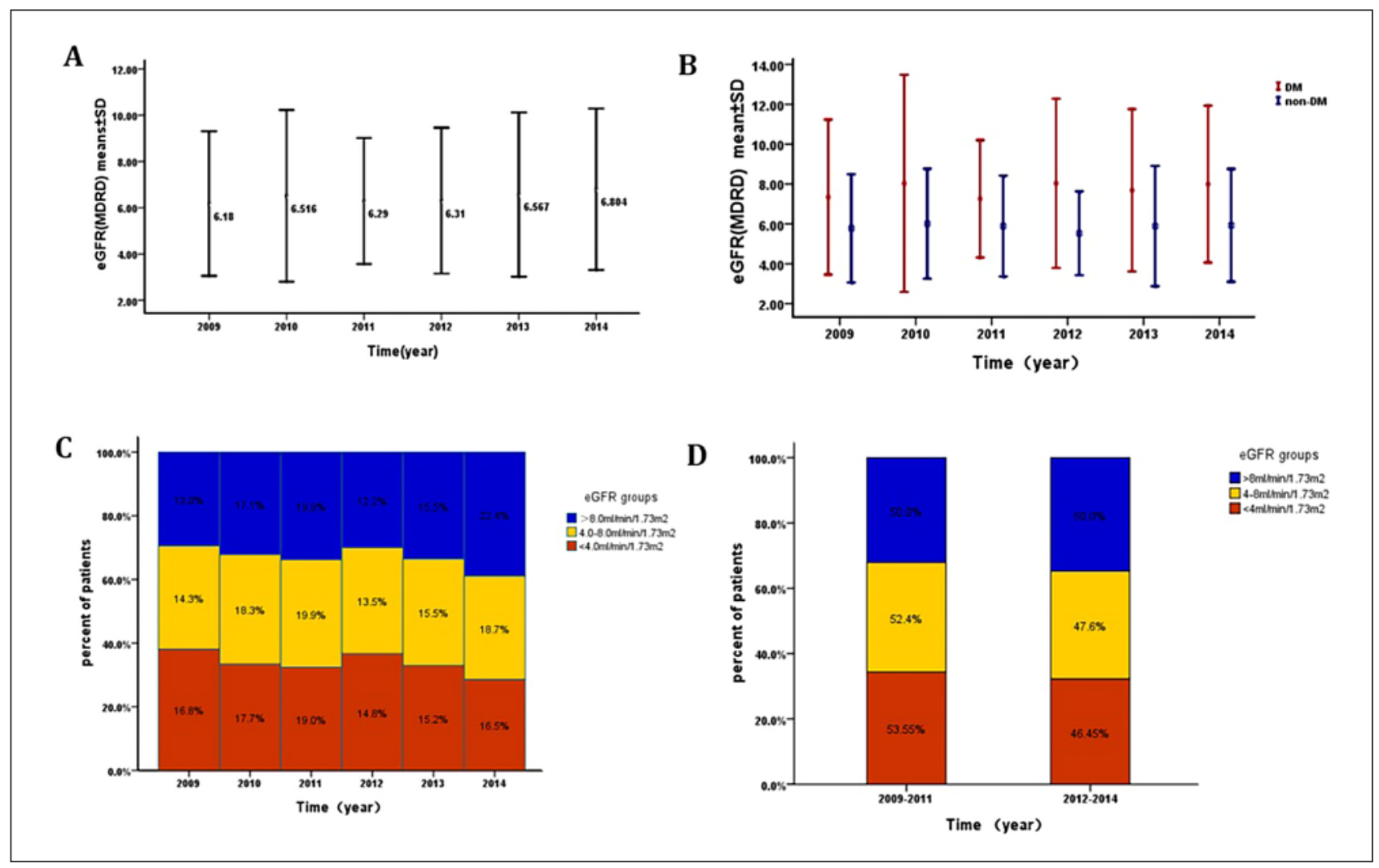

Fig. 2. Change in eGFR at the start of dialysis over time. A The change in eGFR at the start of dialysis yearly from 2008 to 2014. B The change in eGFR yearly at the start of dialysis for patients with diabetes or without diabetes. C Trends in the distribution of eGFR (\%) among patients who initiated hemodialysis from 2009 to 2014 by eGFR groups $\left(<4,4-8\right.$, and $\left.>8 \mathrm{~mL} / \mathrm{min} / 1.73 \mathrm{~m}^{2}\right)$. D Comparison of the proportion of the patients who initiated hemodialysis between $2009-2011$ and 2012-2014 by eGFR groups (<4, 4-8, and >8 mL/ $\min / 1.73 \mathrm{~m}^{2}$ ). eGFR, estimated glomerular filtration.

Table 3. Risk factors for the timing of dialysis initiation associated with eGFR

\begin{tabular}{|c|c|c|c|c|}
\hline \multirow[t]{2}{*}{ Variables } & \multirow[t]{2}{*}{ OR } & \multicolumn{2}{|c|}{ eGFR $>8$ vs. $<4 \mathrm{~mL} / \mathrm{min} / 1.73 \mathrm{~m}^{2}$} & \multirow[t]{2}{*}{$p$ value } \\
\hline & & $95 \%$ CI lower & $95 \%$ CI upper & \\
\hline Gender, male (female as reference) & 3.649 & 2.474 & 5.381 & $<0.001$ \\
\hline \multicolumn{5}{|l|}{ Age, years $(<55$ years old as reference $)$} \\
\hline $55-70$ years & 1.519 & 1.014 & 2.275 & 0.043 \\
\hline$>70$ years & 1.587 & 0.812 & 3.100 & 0.177 \\
\hline Diabetes & 4.589 & 2.953 & 7.131 & $<0.001$ \\
\hline \multicolumn{5}{|l|}{ Clinical signs and/or symptoms at the start of dialysis } \\
\hline Heart failure & 1.743 & 1.168 & 2.601 & 0.007 \\
\hline Symptoms of digestive tract disease & 0.499 & 0.336 & 0.741 & 0.001 \\
\hline Edema of stage II or above & 1.043 & 0.706 & 1.541 & 0.831 \\
\hline Uremia encephalopathy & 1.118 & 0.494 & 2.532 & 0.788 \\
\hline Albumin $\leq 35 \mathrm{~g} / \mathrm{L}(\mathrm{R}$ as $>35 \mathrm{~g} / \mathrm{L})$ & 0.959 & 0.658 & 1.398 & 0.828 \\
\hline Potassium $\geq 6.5 \mathrm{mmol} / \mathrm{L}(\mathrm{R}$ as $<6.5 \mathrm{mmol} / \mathrm{L})$ & 0.655 & 0.267 & 1.606 & 0.355 \\
\hline Phosphorus & 0.046 & 0.032 & 0.067 & $<0.001$ \\
\hline
\end{tabular}

The group with an eGFR $<4 \mathrm{~mL} / \mathrm{min} / 1.73 \mathrm{~m}^{2}$ was used as the reference group. eGFR, estimated glomerular filtration rate. 


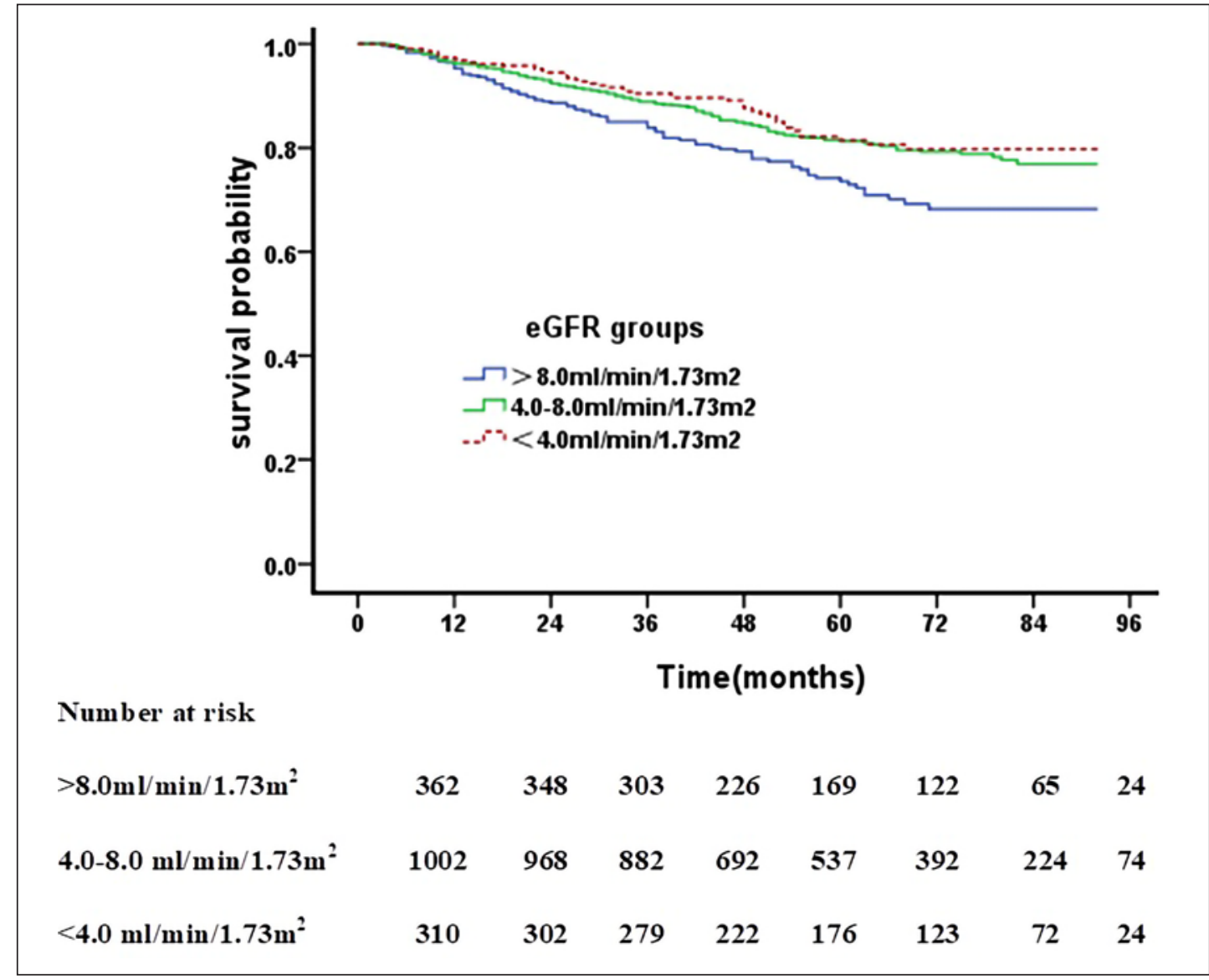

Fig. 3. Kaplan-Meier survival curves of groups based on categories of eGFR $\left(\mathrm{mL} / \mathrm{min} / 1.73 \mathrm{~m}^{2}\right)$ at dialysis initiation $\left(<4,4-8\right.$, and $\left.>8 \mathrm{~mL} / \mathrm{min} / 1.73 \mathrm{~m}^{2}\right)$. Survival rate in the late start group with eGFR $>8 \mathrm{~mL} / \mathrm{min} / 1.73$ $\mathrm{m}^{2}$ was lower than that for the other groups (log-rank test $\left.p=0.002\right)$; there was no significant difference in the survival rate for the group with an eGFR of $4-8 \mathrm{~mL} / \mathrm{min} / 1.73 \mathrm{~m}^{2}$ and the group with an eGFR $<4 \mathrm{~mL} /$ $\min / 1.73 \mathrm{~m}^{2}$ (log-rank test $p=0.478$ ). eGFR, estimated glomerular filtration rate.

\section{Survival Analysis}

Unadjusted Kaplan-Meier analysis suggested that commencing dialysis early was associated with the lowest survival rate (log-rank test $p=0.002$; Fig. 3). However, after adjusting for effectors of age $(<55,55-70,>70)$, gender, diabetes, type of vascular access at initiation, clinical signs, and/or symptoms at the initiation of dialysis (heart failure, symptoms of digestive tract disease, edema of stage II or above, and uremia encephalopathy), and serum albumin $(\leq 35,>35 \mathrm{~g} / \mathrm{L})$, there was no significant difference in survival rate between the 3 groups $\left(<4 \mathrm{~mL} / \mathrm{min} / 1.73 \mathrm{~m}^{2}\right.$ was used as the reference, in comparison with $4-8 \mathrm{~mL} / \mathrm{min} / 1.73$ $\mathrm{m}^{2}[p=0.681]$ and $\left.>8 \mathrm{~mL} / \mathrm{min} / 1.73 \mathrm{~m}^{2}[p=0.403]\right)$.

The Cox regression model demonstrated that old age, diabetes, hypoalbuminemia, presence of uremia encephalopathy before dialysis were risk factors associated with mortality after initiation of dialysis (50-70 years old, hazard ratio [HR] 1.719, 95\% CI [1.323-2.235], $p<0.001 ;>70$ years old, HR 2.868, 95\% CI [2.036-4.040], $p<0.001$; diabetes, HR 1.334, 95\% CI [1.028-1.729], $p=0.030$; hypoalbuminemia, HR 1.471, 95\% CI [1.1641.859], $p=0.001$; uremia encephalopathy, HR 1.817, 95\% CI [1.201-2.750], $p=0.005$; Fig. 4). 


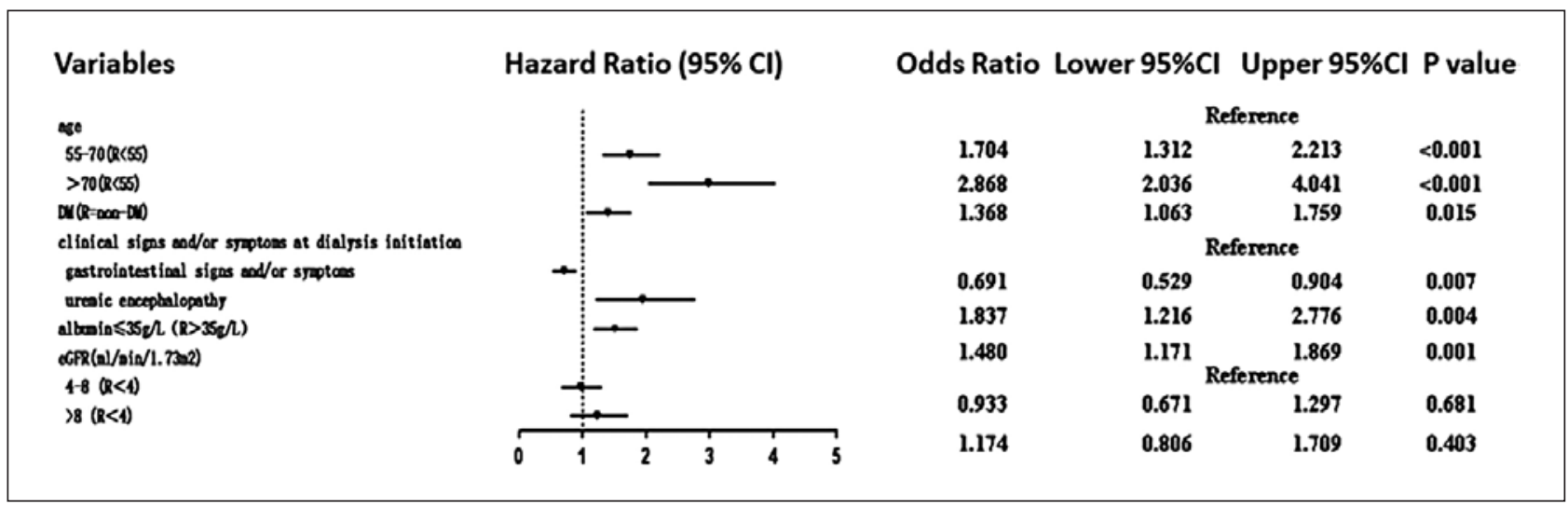

Fig. 4. Adjusted HR of the initiation of dialysis for mortality using a Cox proportional analysis. HR, hazard ratio; DM, diabetes mellitus; eGFR, estimated glomerular filtration rate.

\section{Discussion}

Our study is the first report of nationwide retrospective cohort research concerning the timing of initiation of hemodialysis in patients with ESRD in Mainland China. We analyzed the data for 1647 ESRD patients who started maintenance hemodialysis between 2009 and 2014 . The participating centers were distributed across 6 administrative regions that have high population densities. Therefore, the cohort could represent the timing of dialysis initiation of patients with ESRD between 2009 and 2014 in Mainland China.

The average and median eGFR at the start of dialysis for patients who started dialysis between 2009 and 2014 were $6.5 \pm 3.3$ and $5.8 \mathrm{~mL} / \mathrm{min} / 1.73 \mathrm{~m}^{2}$, respectively, which are lower than those of America, Canada, and the United Kingdom [14, 30-32] while higher than those of Korea, Japan, Singapore, and other countries in East Asia during the same period [15-17]. A previous study reported that the average and median eGFRs at the start of hemodialysis for patients in the Beijing area between 2007 and 2012 were $8.6 \pm 13.0$ and $6.0 \mathrm{~mL} / \mathrm{min} / 1.73 \mathrm{~m}^{2}$ [33], respectively, which are higher than the results shown in our study. However, that study included patients who commenced dialysis with a high eGFR under unstable conditions, such as acute left heart failure, hyperkalemia, and so on, which may lead to the relatively high average eGFR. Meanwhile, that study only included data from one city. In addition, Beijing, as the capital city of China, has a more developed economy and relatively higher medical insurance coverage compared with other areas of China, which could also have contributed to the higher eGFR at the initiation of dialysis compared with that reported in the present study.

There has been a pervasive upward trend of eGFR at initiation within and outside the United States for more than a decade, as the clinical practice guidelines recommended dialysis initiation at a higher GFR [34-37]. After the publication of several observational studies and the IDEAL study, guidelines for dialysis initiation updated after 2012 avoided mentioning a specific eGFR at initiation and thus the trend for early dialysis slowed down [3]. We compared the eGFR at initiation of patients with ESRD in China between 2009-2011 and 2012-2014. However, the results demonstrated that the eGFR at the start of dialysis did not change over time in China, and the trend of eGFR at initiation also did not change after renewal of the guidelines. These results showed that in clinical practice, the timing of dialysis initiation is not dependent on eGFR alone. Meanwhile, the proportion of patients with diabetes between 2012 and 2014 increased compared with that between 2009 and 2011, which is probably related to the increased incidence of diabetes in China in recent years. Subgroup analysis found that patients with ESRD patients and diabetes were more prone to fluid overload, which can lead to heart failure, edema of stage II or 


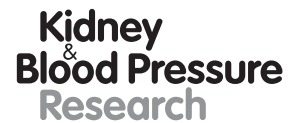

Kidney
Blood Pressure
Research \begin{tabular}{l|l}
\hline Kidney Blood Press Res 2020;45:180-193 \\
\hline DOI: 10.1159/000504671 & $\begin{array}{l}\text { @ 2020 The Author(s). Published by S. Karger AG, Basel } \\
\text { www.karger.com/kbr }\end{array}$ \\
\hline
\end{tabular}

Liu et al.: Timing of Dialysis Initiation in ESRD Patients in China

above, and other related clinical symptoms. Therefore, these patients usually started dialysis with a relatively high eGFR because of the clinical signs and/or symptoms mentioned above. Further logistic regression analysis demonstrated that diabetes was associated with early dialysis initiation, along with being male and the presence of the symptoms of heart failure. Meanwhile, symptoms of digestive tract disease and hyperphosphatemia are associated with late dialysis initiation. Therefore, decisions regarding the initiation of dialysis must take into consideration not only the eGFR but also demographic characteristics, clinical signs and/or symptoms, and other laboratory data, regardless of whether the patient has diabetes $[18,33]$.

Our study also found that from 2012 to 2014, the proportion of dialysis patients using a central venous catheter as the access for dialysis initiation increased from 58.0 to $78.1 \%$. Such an increase is mainly caused by the increased ratio of patients with diabetes, heart failure, and fluid overload. By contrast, the use of central venous catheter for the dialysis initiation was only 18\% in America in 2014, as reported by the 2018 USRDS annual report [38].

By contrast, the use of hemodialysis catheters as the main vascular access for Chinese patients with ESRD at the start of their dialysis indicated that a large proportion of patients had missed their optimal timing of dialysis initiation when they were admitted to hospital, which is possibly another reason why the eGFR at the start of dialysis is lower in Chinese patients ESRD than that in European and American patients. Therefore, it is necessary to explore the optimal timing for dialysis initiation for patients with ESRD in China, which could be assisted by strengthening the follow-up of CKD, improving the education of patients with ESRD patients before dialysis, and better preparation of vascular access.

We found that the proportion of patients with older age, who were male, or who had diabetes was significantly higher in the group with higher eGFR ( $>8 \mathrm{~mL} / \mathrm{min} / 1.73 \mathrm{~m}^{2}$ ) than that in the group with lower eGFR $\left(4-8\right.$ and $\left.<4 \mathrm{~mL} / \mathrm{min} / 1.73 \mathrm{~m}^{2}\right)$. This result is consistent with previous observational studies using large samples [39-41].

This study is also reflected in clinical practice. For example, elderly patients tend to have more complications and need early dialysis initiation. Male patients start dialysis earlier than female patients, possibly because of the presence of more complications in male patients. However, such a difference between the genders could also reflect the use of Modification of Diet in Renal Disease formula 7 for GFR estimation, which is based on the level of blood creatinine. Women have a relatively lower level of creatinine; therefore, the use of this formula could overestimate the renal function in women and ultimately result in late dialysis.

The relationship between eGFR and survival in patients with different timings of dialysis initiation was also analyzed. Using Kaplan-Meier survival curves, we found that the survival rate was significantly lower in patients with a high eGFR at the start of dialysis than in patients with a low eGFR at the start of dialysis. However, such difference disappeared after adjusting for confounding factors, including age, gender, diabetes, clinical signs and/or symptoms (heart failure, symptoms of digestive tract disease, edema of stage II or above, and uremia encephalopathy), and serum albumin. This result is consistent with the most recent observational studies and the IDEAL study [9, 30, 36, 40].

However, all these results are based on the use of eGFR as the only indicator for the analysis of the timing of the dialysis initiation and therefore can only suggest that the eGFR at the start of dialysis was not associated with the mortality of the patients.

In clinical practice, decisions on the timing of dialysis initiation not only consider the patients' renal function but also should take into consideration of the patients' clinical signs and/or symptoms, and nutritional status [18]. Therefore, evaluation using eGFR only to determine the timing of dialysis initiation is not appropriate. In addition, as the clinical signs and/or symptoms, and nutritional status depend on subjective judgment and are difficult to standardize, further research is required to explore a new, quantitative method that uses multiple factors to evaluate the timing of dialysis initiation. 


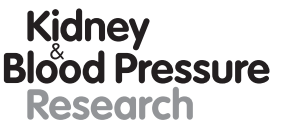

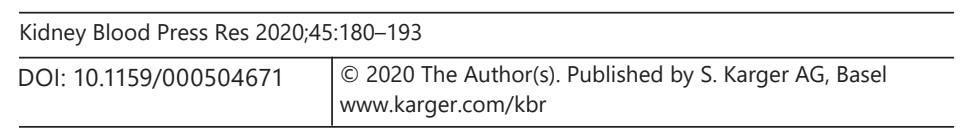

Liu et al.: Timing of Dialysis Initiation in ESRD Patients in China

\section{Conclusion}

From 2009 to 2014, eGFR at the initiation of dialysis for patients with ESRD on maintenance hemodialysis did not change significantly overtime in Mainland China. eGFR at the initiation of dialysis had no association with the survival rate of the patients after dialysis. However, old age, diabetes, uremia encephalopathy, and hypoalbuminemia were the independent risk factors for mortality.

\section{Acknowledgments}

We are grateful to all the patients who participated in this study and to the investigators for their cooperation. The authors thank Dr. Mark Roger Marshall (Associate Professor of University of Auckland, New Zealand) and Dr. Yushan Wei (The First Affiliated Hospital of Dalian Medical University, Dalian, China) for their helpful comments. This study was performed with the support of the special fund for NHFPC Scientific Research in the Public Welfare (201502023) and Liaoning Province Translational Medicine Research Center of Kidney Disease (No. 2014225018). The sponsor had no role in the design and conduction of the study, in the collection, management, analysis, and interpretation of the data, or in the preparation, review, or approval of the manuscript.

\section{Disclosure Statement}

All authors declared no conflicting interests.

\section{Author Contributions}

H.L., X.C., and Y.L.: research idea and study design. Y.L., X.H., and L.W.: data acquisition. All authors: data analysis and interpretation. Y.L., L.W., and H.L.: drafting the manuscript. Y.W., L.W., and Y.L.: statistical analysis. H.L.: study supervision. All authors: contributed to collecting the data on site. Dr. Hongli Lin has full access to all the data of the study and takes responsibility for the integrity of the data and the accuracy of the data analysis.

\section{Appendix}

Investigators of the Dialysis Initiation based on Fuzzy mathematics Equation study group: Hongli Lin, MD, PhD, Ying Liu, MD, the First Affiliated Hospital of Dalian Medical University, Dalian, China. Xuefeng Sun, MD, PhD, Yong Wang MD, Chinese PLA General Hospital, Beijing, China. Hongli Jiang, MD, PhD, Meng Wang, MM, The First Affiliated Hospital of Xi'an Jiaotong University, Xi'an, China. Wei Shi, MD, PhD, Xinling Liang, MD, PhD, Guangdong General Hospital, Guangdong Academy of Medical Sciences, Guangzhou, China. Wenhu Liu, MD, PhD, Beijing Friendship Hospital, Capital Medical University. Ping Fu, MD, PhD, Li Zhou, MD, PhD, West China Hospital of Sichuan University, Chengdu, China. Xiaoqiang Ding, MD, PhD, Jianzhou Zou, MD, Zhongshan Hospital, Fudan University, Shanghai, China. Ming Chang, MM, Dalian Municipal Central Hospital, Dalian, China. Xueqing Yu, MD, PhD, Zhihua Zheng, MD, PhD, The First Affiliated Hospital, Sun Yat-sen University, Guangzhou, China. Ning Cao, PhD, Xiaoling Zhang, MM, General Hospital of Shenyang Military Area Command, Shenyang, 


\section{Kidney \\ Blood Pressure Research}

\begin{tabular}{l|l}
\hline Kidney Blood Press Res 2020;45:180-193 \\
\hline DOI: 10.1159/000504671 & $\begin{array}{l}\text { @ 2020 The Author(s). Published by S. Karger AG, Basel } \\
\text { www.karger.com/kbr }\end{array}$ \\
\hline
\end{tabular}

Liu et al.: Timing of Dialysis Initiation in ESRD Patients in China

China. Menghua Chen, PhD, Zhihong Feng, MD, General Hospital of Ningxia Medial University, Yinchuan, China. Zhaohui Ni, MD, PhD, Mingli Zhu, MD, Renji Hospital, School of Medicine, Shanghai Jiaotong University, Shanghai, China. Jing Chen, MD, PhD, Minmin Zhang, MD, PhD, Huashan Hospital, Fudan University, Shanghai, China. Shiren Sun, PhD, Yan Yu, MM, Xijing Hospital, The Fourth Military Medical University, Xi'an, China. Huimin Wang, MM, Wei Wei, MM, General Hospital of Benxi Iron and Steel Co., Ltd, Benxi, China. Yunyan Wang, BSM, Jie Yang, BSM, Daping Hospital and Surgery Institute, The Third Military Medical University, Chongqing, China. Bihu Gao, PhD, Hua Wu, BSM, Affiliated Zhong Shan Hospital of Dalian University, Dalian, China. Jianqin Wang, PhD, Wenge Wang, BSM, Lanzhou University Second Hospital, Lanzhou, China. Lirong Hao, PhD, Yang Zhou, MD, the First Affiliated Hospital of Harbin Medical University, Harbin, China. Suhua Li, MD, Yingping Sun, MM, The First Affiliated Hospital of Xinjiang Medical University, Urumchi, China. Qiang He, PhD, Xiaogang Shen, MD, Zhejiang Provincial People's Hospital, Hangzhou, China. Hongmei Liu, BSM, Xianghui Kong, MM, An Steel Group Hospital, Anshan, China. Fengmin Shao, PhD, Xiaogang Shen, MD, Henan Provincial People's Hospital and The People's Hospital of Zhengzhou University, Zhengzhou, China. Wei Li, PhD, Yang Wang, MPH, Fuwai Hospital, National Center for Cardiovascular Disease, Peking Union Medical College and Chinese Academy of Medical Sciences, Beijing, China. Ximing Sun, PhD, Degang Wang, PhD, School of Control Science and Engineering, Dalian University of Technology, Dalian, China.

\section{References}

1 Liu ZH. Nephrology in china. Nat Rev Nephrol. 2013 Sep;9(9):523-8.

2 Xie F, Zhang D, Wu J, Zhang Y, Yang Q, Sun X, et al. Design and implementation of the first nationwide, webbased Chinese Renal Data System (CNRDS). BMC Med Inform Decis Mak. 2012 Feb;12(1):11.

3 Rivara MB, Mehrotra R. Timing of Dialysis Initiation: What Has Changed Since IDEAL? Semin Nephrol. 2017 Mar;37(2):181-93.

4 Saggi SJ, Allon M, Bernardini J, Kalantar-Zadeh K, Shaffer R, Mehrotra R; Dialysis Advisory Group of the American Society of Nephrology. Considerations in the optimal preparation of patients for dialysis. Nat Rev Nephrol. 2012 Apr;8(7):381-9.

5 Mehrotra R, Rivara M, Himmelfarb J. Initiation of dialysis should be timely: neither early nor late. Semin Dial. 2013 Nov-Dec;26(6):644-9.

6 Bonomini V, Feletti C, Scolari MP, Stefoni S. Benefits of early initiation of dialysis. Kidney Int Suppl. 1985 Dec; 17:S57-9.

7 Hakim RM, Lazarus JM. Initiation of dialysis. J Am Soc Nephrol. 1995 Nov;6(5):1319-28.

8 Korevaar JC, Jansen MA, Dekker FW, Jager KJ, Boeschoten EW, Krediet RT, et al.; Netherlands Cooperative Study on the Adequacy of Dialysis Study Group. When to initiate dialysis: effect of proposed US guidelines on survival. Lancet. 2001 Sep;358(9287):1046-50.

9 Cooper BA, Branley P, Bulfone L, Collins JF, Craig JC, Fraenkel MB, et al.; IDEAL Study. A randomized, controlled trial of early versus late initiation of dialysis. N Engl J Med. 2010 Aug;363(7):609-19.

10 Hwang SJ, Yang WC, Lin MY, Mau LW, Chen HC; Taiwan Society of Nephrology. Impact of the clinical conditions at dialysis initiation on mortality in incident haemodialysis patients: a national cohort study in Taiwan. Nephrol Dial Transplant. 2010 Aug;25(8):2616-24.

11 Crews DC, Scialla JJ, Boulware LE, Navaneethan SD, Nally JV Jr, Liu X, et al.; DEcIDE Network Patient Outcomes in End Stage Renal Disease Study Investigators. Comparative effectiveness of early versus conventional timing of dialysis initiation in advanced CKD. Am J Kidney Dis. 2014 May;63(5):806-15.

12 Scialla JJ, Liu J, Crews DC, Guo H, Bandeen-Roche K, Ephraim PL, et al.; DEcIDE Network Patient Outcomes in End Stage Renal Disease Study Investigators. An instrumental variable approach finds no associated harm or benefit with early dialysis initiation in the United States. Kidney Int. 2014 Oct;86(4):798-809.

13 Yamagata K, Nakai S, Iseki K, Tsubakihara Y; Committee of Renal Data Registry of the Japanese Society for Dialysis Therapy. Late dialysis start did not affect long-term outcome in Japanese dialysis patients: long-term prognosis from Japanese Society for [corrected] Dialysis Therapy Registry. Ther Apher Dial. 2012 Apr;16(2):111-20.

14 Saran R, Li Y, Robinson B, Abbott KC, Agodoa LY, Ayanian J, et al. US Renal Data System 2015 Annual Data Report: Epidemiology of Kidney Disease in the United States. Am J Kidney Dis. 2016 Mar;67(3 Suppl 1):Svii, S1-305.

15 Yamagata K, Nakai S, Masakane I, Hanafusa N, Iseki K, Tsubakihara Y; Committee of Renal Data Registry of the Japanese Society for Dialysis Therapy. Ideal timing and predialysis nephrology care duration for dialysis initiation: from analysis of Japanese dialysis initiation survey. Ther Apher Dial. 2012 Feb;16(1):54-62. 


\section{Kidney \\ Blood Pressure \\ Research}

\begin{tabular}{l|l}
\hline Kidney Blood Press Res 2020;45:180-193 \\
\hline DOI: 10.1159/000504671 & $\begin{array}{l}\text { @ 2020 The Author(s). Published by S. Karger AG, Basel } \\
\text { www.karger.com/kbr }\end{array}$ \\
\hline
\end{tabular}

Liu et al.: Timing of Dialysis Initiation in ESRD Patients in China

16 Feng L, Jin AZ, Allen JC, Chow KY, Jafar TH. Timing of commencement of maintenance dialysis and mortality in young and older adults in Singapore. BMC Nephrol. 2017 May;18(1):176.

17 Lee MJ, Kim S, Park I, Kim H, Shin GT. Comparison of estimated glomerular filtration rate equations at the time of hemodialysis initiation. Kidney Res Clin Pract. 2015 Dec;34(4):207-11.

18 Daugirdas JT, Depner TA, Inrig J, Mehrotra R, Rocco MV, Suri RS, et al.; National Kidney Foundation. 2015 update. Am J Kidney Dis. 2015 Nov;66(5):884-930.

19 Nesrallah GE, Mustafa RA, Clark WF, Bass A, Barnieh L, Hemmelgarn BR, et al. Canadian Society of Nephrology 2014 clinical practice guideline for timing the initiation of chronic dialysis. CMAJ. 2014 Feb; 186(2):112-7.

20 Watanabe Y, Yamagata K, Nishi S, Hirakata H, Hanafusa N, Saito C, et al.; "Hemodialysis Initiation for Maintenance Hemodialysis" Guideline Working Group, Japanese Society for Dialysis Therapy. Japanese society for dialysis therapy clinical guideline for "hemodialysis initiation for maintenance hemodialysis". Ther Apher Dial. 2015 Mar; 19 Suppl 1:93-107.

21 Tattersall J, Dekker F, Heimbürger O, Jager KJ, Lameire N, Lindley E, et al.; ERBP Advisory Board. When to start dialysis: updated guidance following publication of the Initiating Dialysis Early and Late (IDEAL) study. Nephrol Dial Transplant. 2011 Jul;26(7):2082-6.

22 Zhang L, Wang F, Wang L, Wang W, Liu B, Liu J, et al. Prevalence of chronic kidney disease in China: a crosssectional survey. Lancet. 2012 Mar;379(9818):815-22.

23 Xu Y, Wang L, He J, Bi Y, Li M, Wang T, et al.; 2010 China Noncommunicable Disease Surveillance Group. Prevalence and control of diabetes in Chinese adults. JAMA. 2013 Sep;310(9):948-59.

24 Gao Y, Chen G, Tian H, Lin L, Lu J, Weng J, et al.; China National Diabetes and Metabolic Disorders Study Group. Prevalence of hypertension in china: a cross-sectional study. PLoS One. 2013 Jun;8(6):e65938.

25 Chinese National Renal Data System. at http://www.cnrds.net.

26 Lina Z, Wenlv L, Jie T. Association of residual renal function at imtiation of dialysis with prognosis in maintenance dialysis patients. Chin J Nephrol. 2012;28:757-64.

27 Li L, Mei W, Xuemei L. The trend of the timing at which hemodialysis initiated in Beijing area. Chin J Blood Purif. 2014;12:5.

28 Kidney Disease: improving Global Outcomes (KDIGO) CKD Work Group. KDIGO clinical practice guideline for the evaluation and management of chronic kidney disease. Kidney Int Suppl. 2013;3:1-150.

29 Levey AS, Bosch JP, Lewis JB, Greene T, Rogers N, Roth D; Modification of Diet in Renal Disease Study Group. A more accurate method to estimate glomerular filtration rate from serum creatinine: a new prediction equation. Ann Intern Med. 1999 Mar;130(6):461-70.

30 O'Hare AM, Wong SP, Yu MK, Wynar B, Perkins M, Liu CF, et al. Trends in the Timing and Clinical Context of Maintenance Dialysis Initiation. J Am Soc Nephrol. 2015 Aug;26(8):1975-81.

31 Yu MK, O’Hare AM, Batten A, Sulc CA, Neely EL, Liu CF, et al. Trends in Timing of Dialysis Initiation within Versus Outside the Department of Veterans Affairs. Clin J Am Soc Nephrol. 2015 Aug;10(8):1418-27.

32 Stel VS, Dekker FW, Ansell D, Augustijn H, Casino FG, Collart F, et al. Residual renal function at the start of dialysis and clinical outcomes. Nephrol Dial Transplant. 2009 Oct;24(10):3175-82.

33 Rivara MB, Chen CH, Nair A, Cobb D, Himmelfarb J, Mehrotra R. Indication for Dialysis Initiation and Mortality in Patients With Chronic Kidney Failure: A Retrospective Cohort Study. Am J Kidney Dis. 2017 Jan;69(1): 41-50.

34 Rosansky SJ, Clark WF, Eggers P, Glassock RJ. Initiation of dialysis at higher GFRs: is the apparent rising tide of early dialysis harmful or helpful? Kidney Int. 2009 Aug;76(3):257-61.

35 Clark WF, Na Y, Rosansky SJ, Sontrop JM, Macnab JJ, Glassock RJ, et al. Association between estimated glomerular filtration rate at initiation of dialysis and mortality. CMAJ. 2011 Jan 11;183(1):47-53.

36 O'Hare AM, Choi AI, Boscardin WJ, Clinton WL, Zawadzki I, Hebert PL, et al. Trends in timing of initiation of chronic dialysis in the United States. Arch Intern Med. 2011 Oct;171(18):1663-9.

37 Couchoud C, Guihenneuc C, Bayer F, Stengel B; REIN Registry. The timing of dialysis initiation affects the incidence of renal replacement therapy. Nephrol Dial Transplant. 2010 May;25(5):1576-8.

38 Saran R, Robinson B, Abbott KC, Agodoa LY, Albertus P, Ayanian J, et al. US Renal Data System 2016 Annual Data Report: Epidemiology of Kidney Disease in the United States. Am J Kidney Dis. 2017 Mar;69(3 Suppl 1):A7-8.

39 Kausz AT, Obrador GT, Arora P, Ruthazer R, Levey AS, Pereira BJ. Late initiation of dialysis among women and ethnic minorities in the United States. J Am Soc Nephrol. 2000 Dec;11(12):2351-7.

40 Wright S, Klausner D, Baird B, Williams ME, Steinman T, Tang H, et al. Timing of dialysis initiation and survival in ESRD. Clin J Am Soc Nephrol. 2010 Oct;5(10):1828-35.

41 Rosansky SJ, Eggers P, Jackson K, Glassock R, Clark WF. Early start of hemodialysis may be harmful. Arch Intern Med. 2011 Mar;171(5):396-403. 